\title{
RNA interference and retinoblastoma-related genes are required for repression of endogenous siRNA targets in Caenorhabditis elegans
}

\author{
Alla Grishok ${ }^{\mathrm{a}, 1}$, Sebastian Hoersch ${ }^{\mathrm{a}, \mathrm{b}}$, and Phillip A. Sharpa,2 \\ ${ }^{a}$ Koch Institute for Integrative Cancer Research, Massachusetts Institute of Technology, Cambridge, MA 02139; and bioinformatics Group, \\ Max Delbrück Center for Molecular Medicine, 13125 Berlin, Germany \\ Contributed by Phillip A. Sharp, October 23, 2008 (sent for review August 15, 2008)
}

In Caenorhabditis elegans, a vast number of endogenous short RNAs corresponding to thousands of genes have been discovered recently. This finding suggests that these short interfering RNAs (siRNAs) may contribute to regulation of many developmental and other signaling pathways in addition to silencing viruses and transposons. Here, we present a microarray analysis of gene expression in RNA interference (RNAi)-related mutants rde-4, zfp-1, and alg-1 and the retinoblastoma (Rb) mutant lin-35. We found that a component of Dicer complex RDE-4 and a chromatin-related zinc finger protein ZFP-1, not implicated in endogenous RNAi, regulate overlapping sets of genes. Notably, genes a) up-regulated in the rde-4 and zfp-1 mutants and b) up-regulated in the lin-35(Rb) mutant, but not the down-regulated genes are highly represented in the set of genes with corresponding endogenous siRNAs (endosiRNAs). Our study suggests that endogenous siRNAs cooperate with chromatin factors, either C. elegans ortholog of acute lymphoblastic leukemia-1 (ALL-1)-fused gene from chromosome 10 (AF10), ZFP-1, or tumor suppressor Rb, to regulate overlapping sets of genes and predicts a large role for RNAi-based chromatin silencing in control of gene expression in C. elegans.

endo-siRNA | microarray $\mid \mathrm{Rb}$ | RNAi

A mong the species with sequenced genomes the nematode Caenorhabditis elegans encodes the largest number of Argonaute proteins, which interact with short RNAs (1). Also, a large number of endogenous, short interfering RNAs (endo-siRNAs) have been cloned from $C$. elegans (2-5). They are distinct from microRNAs (miRNAs), are largely generated by RNA-dependent RNA polymerases (RdRP), and match thousands of genes. These observations suggest that multiple gene-regulatory networks involving Argonaute proteins and endo-siRNAs exist in the nematode.

We have characterized a system of RNAi-induced transcriptional gene silencing (RNAi-TGS) of a repetitive transgene expressed in the soma of C. elegans (6). Also, we found that RNAi pathway genes and lin-35( $\mathrm{Rb})$ synergize in repressing the intestinal cell divisions and in repressing the cyclin E gene (cye-1) expression, likely through cooperative inhibition of cye-1 transcription (7). Two chromatinrelated genes, $z f p-1$ and $g f l-1$, promote the RNAi process in $C$. elegans, either directly or indirectly, they also contribute to RNAiTGS of a repetitive transgene $(6,8,9)$. Interestingly, both genes were also found to antagonize the repressive function of LIN$35(\mathrm{Rb})(10,11)$. Therefore, ZFP-1 and GFL-1 appear to regulate both RNAi and Rb target genes.

The C. elegans Rb protein LIN-35 represses inappropriate transcription of germline-specific genes (12) and growth factors (13) in differentiated somatic cells and functions redundantly with other transcriptional repressors (14). Also, lin-35 mutants are more sensitive to exogenous RNAi than wild-type worms $(11,15)$. This might be partially because of the de-repression of germline-specific RNAi pathway genes in somatic cells.

Because RNAi genes were found to function in the same processes as lin-35, we conducted microarray experiments to find potential targets regulated by RNAi-TGS and lin-35. We used $r d e-4$ and $z f p-1$ mutants affecting RNAi-TGS. RDE-4 is a dsRNA binding protein interacting with Dicer (16) whereas ZFP-1 is a nuclear protein that is likely to affect transcription directly. Our previous study indicated that miRNAs might have a role in promoting RNAi-TGS in C. elegans as well (6); therefore, we included miRNA pathway Argonaute mutant alg-1 in our experiments.

Our analysis revealed $i$ ) that $z f p-1$ and $r d e-4$ mutant animals have strikingly similar profiles of alterations in gene expression and $i i$ ) that there is an enrichment of genes with matching (antisense) endo-siRNAs (3-5) only among genes up-regulated, but not downregulated, in $z f p-1$ and $r d e-4$ mutants. These genes therefore might represent direct targets of chromatin-based silencing induced by endogenous RNAi pathways. Interestingly, endo-siRNAs matched not only genes negatively regulated by $r d e-4$ and $z f p-1$, but also those primarily inhibited by LIN-35(Rb).

We also report that $z f p-1$, unlike $r d e-4$, opposes the repressive function of LIN-35 in controlling intestinal nuclear divisions and cye- 1 expression. Our results suggest that ZFP-1 may play both a positive and a negative role in regulating gene expression.

\section{Results}

Microarray Data Analysis. To find target genes regulated by RNAi and $\mathrm{Rb}$, we performed a series of microarray experiments using RNA from L1-L2 larvae of the wild type and loss-of-function mutants rde-4 (17), zfp-1 (10), alg-1 (7), and lin-35 (18). We conducted pairwise comparisons of the levels of gene expression in each mutant compared with the wild type and selected statistically significant changes in gene expression by two-sample $t$ test ( $P$ value $<0.01$ ), requiring in addition an expression difference of at least 1.5 -fold between two group averages. Our microarray data are summarized in Dataset S1 and Dataset S2.

A majority of the genes changing expression in the lin-35 mutant compared with the wild type (535 of 710 ) were up-regulated consistent with the repressive role of the LIN-35 protein (Table 1). Similar numbers of genes were either up-regulated or downregulated in each of the RNAi-related mutants: 420 were "up" in $z f p-1$ and 434 were "down" whereas 285 were "up" in $r d e-4$ and 219 were "down", and 170 were "up" in alg-1 and 213 were "down." The numbers of genes similarly regulated in different mutants are listed in Table 1. Ten genes commonly up-regulated in all four mutants are described in Table S1.

Author contributions: A.G., S.H., and P.A.S. designed research; A.G. performed research; A.G. and S.H. analyzed data; and A.G., S.H., and P.A.S. wrote the paper.

The authors declare no conflict of interest.

Data deposition: The data reported in this paper have been deposited in the Gene Expression Omnibus (GEO) database, www.ncbi.nlm.nih.gov/geo (accession no. GSE13258).

1Present address: Department of Biochemistry and Molecular Biophysics, College of Physicians and Surgeons, Columbia University, New York, NY 10032.

${ }^{2}$ To whom correspondence should be addressed at: Koch Institute for Integrative Cancer Research, MIT, 40 Ames Street, E17-529, Cambridge, MA 02139. E-mail: sharppa@mit.edu.

This article contains supporting information online at www.pnas.org/cgi/content/full/ 0810589105/DCSupplemental.

C 2008 by The National Academy of Sciences of the USA 
Table 1. Numbers of genes changing expression compared with the wild type in indicated mutant backgrounds (top) and numbers of overlapping genes between indicated mutants (bottom)

\begin{tabular}{|c|c|c|c|c|c|c|}
\hline Mutant backgrounds & \# genes UP & Enrichment & $P$ value & \# genes DOWN & Enrichment & $P$ value \\
\hline $\operatorname{lin}-35$ & 535 & & & 175 & & \\
\hline$z f p-1$ & 420 & & & 434 & & \\
\hline rde-4 & 285 & & & 219 & & \\
\hline alg-1 & 170 & & & 213 & & \\
\hline lin-35 and $z f p-1$ & 56 & 4.54 & $1.39 \times 10^{-21}$ & 65 & 15.59 & $2.15 \times 10^{-60}$ \\
\hline lin-35 and rde-4 & 40 & 4.78 & $2.07 \times 10^{-16}$ & 32 & 15.21 & $9.1 \times 10^{-29}$ \\
\hline $\operatorname{lin}-35$ and $a \lg -1$ & 39 & 7.81 & $5.45 \times 10^{-24}$ & 40 & 19.55 & $1.58 \times 10^{-40}$ \\
\hline$z f p-1$ and $r d e-4$ & 110 & 16.74 & $1.44 \times 10^{-107}$ & 138 & 26.45 & $2.78 \times 10^{-174}$ \\
\hline$z f p-1$ and $a l g-1$ & 68 & 17.35 & $7.26 \times 10^{-67}$ & 131 & 25.81 & $7.40 \times 10^{-163}$ \\
\hline rde-4 and alg-1 & 41 & 15.41 & $3.77 \times 10^{-37}$ & 77 & 30.07 & $2.65 \times 10^{-96}$ \\
\hline
\end{tabular}

Genes up-regulated in the mutants are listed in the "UP" column and down-regulated gene are listed in the "DOWN" column. Enrichment factors and $P$ values

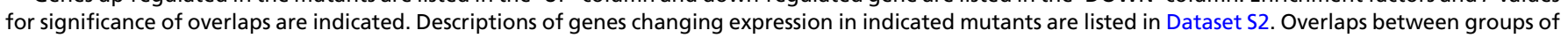

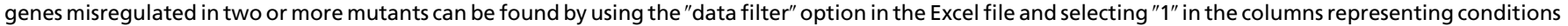
of interest.

zfp-1 and rde-4 Mutants Have Similar Gene Expression Profiles. A comparison of gene sets misregulated in the studied mutants revealed a very significant overlap between genes regulated by $r d e-4$ and genes regulated by $z f p-1$, including genes both up-regulated and down-regulated in the mutants compared with wild type (Table 1). Fifty percent of genes regulated by rde-4 (close to 250) are included in a group affected by $z f p-1$. This degree of overlap in transcriptome regulation has not been reported before for any pair of RNAirelated genes.

Next, we used the gene expression terrain map ("topomap") (19) as a platform for functional annotation of misregulated gene sets. In this work, based on the analysis of extensive microarray expression datasets, 17,658 C. elegans genes were divided into forty-five expression clusters ("mounts") of coregulated genes. Kim and colleagues also redundantly assigned membership in 56 functional categories to 5,615 functionally characterized $C$. elegans genes, resulting in 8,212 category assignments (19). We mapped our datasets of misregulated genes in various mutants to mounts and categories (Fig. 1). A heatmap representation with clustering dendrograms summarizing significant enrichment of genes from ours and other relevant studies in functional groups of genes (mounts and categories) defined by Kim and colleagues (19) is shown in Fig. 1 and, more completely, in Fig. S1. In this representation, related functional groups are clustered on the $y$ axis and related datasets are clustered on the $x$ axis. This allows functional annotation and comparison of multiple datasets. P-values for statistical significance and representation factors for gene enrichment in specific groups are listed in Dataset S3.

We chose topomap as a vehicle for functional analysis over possible alternatives, especially gene ontology (GO) annotation, for a number of reasons. Chief among them is the considerably greater coverage of C. elegans genes ( $77 \%$ for topomap compared with $46 \%$ for GO) that is-by nature of the "annotation process"-not restricted to known and characterized genes. Therefore, topomapbased functional assignment described in our study is not limited to well studied genes. Functional annotation of our expression data using GO platform (data not shown) was similar to that obtained with topomap, but we arrived at a more complete picture of gene expression by using topomap.

A comparison of the functional categories of genes changing expression in different mutants revealed a striking similarity between transcriptome profiles in $r d e-4$ and $z f p-1$ mutants (Fig. 1 and Fig. S1). This similarity suggests that common biological processes are affected by both mutations. For example, certain germlineenriched and oocyte genes (mount \#02) are overrepresented in groups of genes with higher expression levels in $z f p-1$ and $r d e-4$ mutants and close to $20 \%$ of genes commonly up-regulated in both mutants belong to this category (Dataset S2). Indeed, functional annotation of the groups of genes commonly affected by each combination of two mutants (presented in Table 1) revealed the same categories of enrichment as those that were common between the two single mutant profiles (Fig. S2).

Therefore, two independent types of analyses: 1) a direct comparison of genes changing expression in two mutants (Table 1) and 2) functional annotation of misregulated genes (Fig. 1 and Figs. S1 and S2) strongly suggest that $z f p-1$ and $r d e-4$ work in the same pathway (RNAi-TGS) and point to a very significant role of this pathway in biology of $C$. elegans.

The $r d e-4$ mRNA level was not changed in the $z f p-1$ mutant and vice versa, indicating that a simple model of regulation of one gene by the product of another does not account for the correlation. We cannot exclude the possibility that protein levels of RDE-4 or ZFP-1 might change. However, these types of changes are not likely to be due to the direct regulation by RDE- 4 or ZFP-1 because RDE-4 is known to interact with RNA and ZFP-1 is a chromatin factor.

Genes with higher expression in $z f p-1$ and $r d e-4$ mutants were overrepresented among the functional groups 'protein expression,' 'germline-enriched,' 'biosynthesis,' 'mitochondrial,' and 'cell cycle,' whereas those genes that were down-regulated in the mutants frequently represented intestine-specific genes involved in metabolic processes (Fig. 1). Histone genes were also significantly enriched in the rde-4 down-regulated gene set (Fig. 1). Importantly, ZFP-1 appears to have a larger role in gene expression regulation than RDE-4 (Fig. 1, Table 1, and Dataset S1). Consistent with these results, $z f p-1$ mutants have some developmental phenotypes, such as slow growth and protruded vulva (10), whereas $r d e-4$ mutant worms are superficially normal.

A recent microarray study reported gene expression changes in the RNAi pathway mutants $r d e-1, r d e-4$, and $d c r-1$ (20). We mapped the misregulated gene sets from this study to the functional groups of coregulated genes (Fig. 1) and found that genes down-regulated in the $r d e-4$ mutant were enriched in intestine-specific group contained significant number of histone genes and proteases. This signature corresponds to that of genes down-regulated in the rde-4 mutant from our study (Fig. 1). However, genes found up-regulated in the rde-4 mutant do not have a signature consistent with our findings (Fig. 1). One difference between the studies is that we used L1-L2 larva and the published report used adult worms (20). Because adult worms contain both differentiated somatic tissues and actively proliferating and specialized germline cells, whereas the L1-L2 larvae contain primarily somatic cells, the resulting "average" gene expression profile is likely to be different in adults and larvae. In addition, mutant backgrounds may have different effects on gene expression in somatic and germline tissues. 


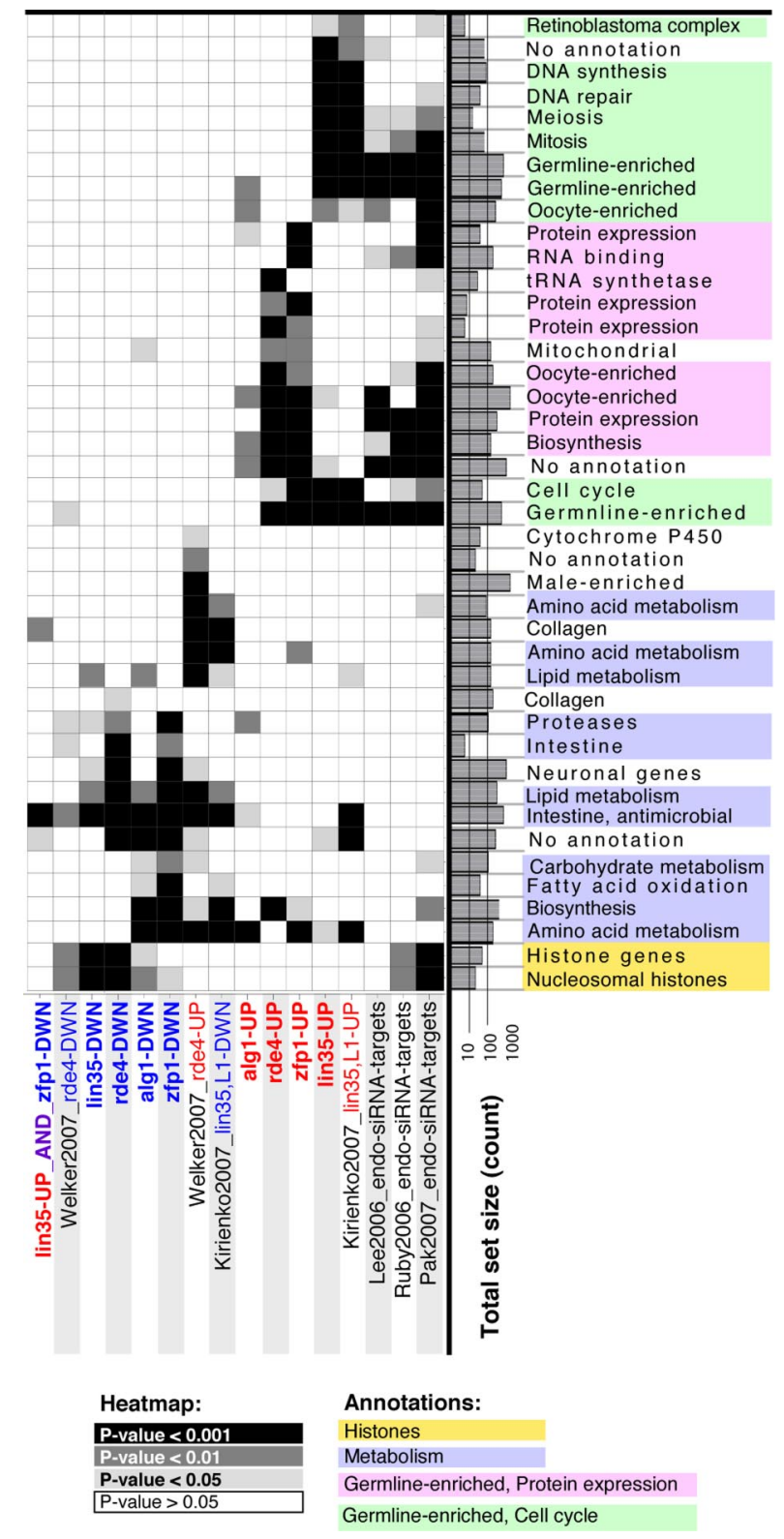

Fig. 1. Heatmap showing the enrichment of selected functional groups as defined by ref. 19 ( $y$ axis) in gene sets from various datasets ( $x$ axis). The gray shades in the heatmap indicate significance levels as indicated in the legend. The bars to the right of the heatmap indicate the total size of each functional group. For a comprehensive version of the figure including all functional groups with any significant enrichment and clustering dendrograms informing the order of groups and datasets, see Fig. S1. Enrichment factors and their $P$ values are presented in Dataset S3.

Endogenous siRNA Preferentially Map to Genes Up-Regulated in zfp-1, rde-4, and lin-35 Mutants. As mentioned, we found almost equal numbers of genes both positively and negatively regulated by RDE-4 and ZFP-1 (Table 1). Although it is conceivable that ZFP-1 might act directly as an activator or as a repressor on both types of target genes, RDE-4, required for the production of siRNAs (21), is much more likely to contribute directly only to gene silencing.

To gain insight about possible direct targets of RNAi-TGS, we mapped the endo-siRNA target genes according to three independent studies (3-5) to the coregulated groups defined by Kim and colleagues (Fig. 1). Interestingly, distribution of siRNA target genes was nonrandom and mostly consistent among the three studies
(3-5) (Fig. 1). We found that siRNA-matching genes were very significantly enriched in the same functional groups as the genes up-regulated in $z f p-1$ and $r d e-4$ mutants or lin-35 mutants (Fig. 1), but not in the groups overrepresented in genes down-regulated in the same mutants, with the exception of histone genes (Fig. 1). This suggested that specific genes negatively regulated by $z f p-1$ and $r d e-4$ or by lin-35 might be more likely to have a matching endo-siRNA.

Indeed, direct comparison of endo-siRNA target gene sets and $z f p-1$, rde-4, alg-1, and lin-35-regulated genes revealed a statistically significant enrichment of genes with siRNAs only in the sets of genes up-regulated in the mutants but not in the down-regulated sets of genes (Table 2). Close to $50 \%$ of genes with increased expression in each of the mutant strains were reported to have a matching siRNA. These data strongly suggest that genes upregulated in the mutants represent the direct targets repressed by RNAi and that the down-regulated genes might be affected by the mutations indirectly. A very large overlap between $r d e-4$ and $z f p$-1-regulated genes (Table 1) together with high significance of enrichment in siRNA targets of gene sets up-regulated in both mutants (Table 2) further strengthens the prediction of numerous target genes regulated by RNAi-TGS in C. elegans.

When functional annotation was done on the groups of genes representing overlaps between genes up-regulated in the mutants and siRNA target genes, the signatures of "UP in $z f p-1$ and siRNA target" and "UP in rde-4 and siRNA target" groups were found to be very similar and very close to "UP in $z f p-1$ and $r d e-4$ " signature. On the contrary, the signature of the "UP in lin-35 and siRNA target" group was distinct from "UP in $z f p-1$ and $r d e-4$ " and very similar to that of "UP in lin-35", whereas "UP in alg-1 and siRNA target" group had similarity to groups of genes regulated by $z f p-1$ and $r d e-4$ and also regulated by lin-35. We conclude that siRNA targets overlapping with lin-35-regulated genes are distinct from the groups of siRNA targets regulated by $z f p-1$ and $r d e-4$. Although chromatin factor ZFP-1 may be directly involved in the endosiRNA pathway as this gene was implicated in supporting RNAi, the overlap between lin-35 and endo-siRNA target genes likely represents synergy between the two repressive pathways.

The main signature of the lin-35 mutant is de-repression of germline-specific genes in somatic tissues of larvae (Fig. 1), which is consistent with previous findings $(11,22)$. There are three main groups of coregulated genes that represent germ line (mounts \#02, \#07, and \#11, Fig. S1). Endogenous siRNAs are enriched in those same groups: of the total of 4,372 siRNA targets represented in the topomap dataset, 1,448 were found to belong to these germline mounts. Direct comparison of germline-specific siRNA target genes with the sets of genes changing expression in the mutants (Table S2) revealed $9 \times$ overrepresentation of genes up-regulated in lin-35 larvae (154 compared with 17 expected by chance). This correlation may indicate that endo-siRNAs synergize with LIN-35 in repressing germline-specific fate in somatic tissues. Alternatively, although both LIN-35 targets and endo-siRNAs preferentially correspond to germline-enriched genes, LIN-35 and RNAi may regulate those genes independently in distinct tissues: soma and germ line, respectively.

When we compared nongermline siRNA target genes with gene sets changing expression in the mutants, genes up-regulated in lin-35 were enriched modestly $(2.5 \times)$ and enriched less than genes down-regulated in lin-35 (3.3×) (Table S3). In contrast, genes up-regulated in $z f p-1$ and $r d e-4$ were overrepresented among siRNA targets $(4 \times)$, independently of their germline or nongermline classification (Tables S2 and S3). The corresponding sets of downregulated genes were not overrepresented. This analysis further supports synergy between endo-siRNAs, $r d e-4$ and $z f p-1$, in gene expression regulation. Although lin-35 and endo-siRNA targets do not appear to correlate outside of germline-enriched group of genes, the possibility of synergy between LIN-35 and endo-siRNAs in repressing germline fate in the soma still remains and needs to be studied further. 
Table 2. endo-siRNA targets are overrepresented among genes up-regulated in RNAi and Rb mutants

\begin{tabular}{|c|c|c|c|c|c|c|c|c|}
\hline & UP in lin-35 & $\begin{array}{l}\text { UP in } \\
z f p-1\end{array}$ & $\begin{array}{l}\text { UP in } \\
\text { rde-4 }\end{array}$ & $\begin{array}{l}\text { UP in } \\
\text { alg-1 }\end{array}$ & $\begin{array}{l}\text { DOWN in } \\
\text { lin-35 }\end{array}$ & $\begin{array}{l}\text { DOWN in } \\
z f p-1\end{array}$ & $\begin{array}{l}\text { DOWN in } \\
\text { rde- } 4\end{array}$ & $\begin{array}{l}\text { DOWN in } \\
\text { alg-1 }\end{array}$ \\
\hline Totals in $18,459 \downarrow \rightarrow$ & 428 & 333 & 232 & 142 & 143 & 371 & 191 & 185 \\
\hline $\begin{array}{l}\text { Endo-siRNA targets } \\
\quad 3,892\end{array}$ & $\begin{array}{c}\text { Overlap with } \\
\text { endo-siRNA targets }\end{array}$ & & & & & & & \\
\hline 3,892 & 223 & 149 & 107 & 61 & 37 & 74 & 41 & 48 \\
\hline Representation $\mathrm{f}-\mathrm{r}$ & 2.5 & 2.1 & 2.2 & 2 & 1.2 & 0.9 & 1 & 1.2 \\
\hline$P$ value & $1.8 \times 10^{-46}$ & $1.4 \times 10^{-22}$ & $9.6 \times 10^{-18}$ & $3.2 \times 10^{-9}$ & 0.09 & 0.73 & 0.48 & 0.064 \\
\hline
\end{tabular}

Endo-siRNA target gene set, according to Pak and Fire (2) and gene sets determined to be "UP"- or "DOWN"-regulated in our expression data were mapped

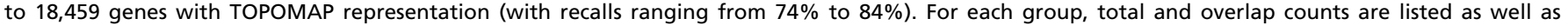
representation factors and $p$ values for overlaps.

Notably, the cyclin E gene targeted by endo-siRNAs is expressed very highly during oogenesis and is categorized as 'germlineenriched.' Therefore, its repression by Rb and RNAi pathways in somatic tissues that we discovered genetically (7) may serve as an example of possible large-scale cooperation between endo-siRNAs and LIN-35 in repressing common targets.

Mutation in zfp-1 Suppresses Extra Nuclei Division Phenotype and Enhanced Cyclin E Expression in lin-35 Mutant Worms. We have found that the combination of the RNAi pathway mutants $r d e-1$, rde-4, or the miRNAi pathway mutants $d c r-1$ and alg-1 with the lin-35 mutation leads to a significant increase in postembryonic nuclear divisions in the intestine of the double mutant worms (Fig. $2 A$ and $B)$ (7). Increases in cyclin $\mathrm{E}$ (cye-1) transcription under these conditions are at least partially responsible for this phenotype (7).

Because $r d e-4$ and $z f p-1$ regulate many common genes, we tested whether ZFP-1 also cooperates with LIN-35 in repressing cye-1. Surprisingly, we found that combining the $z f p-1$ mutation with lin-35(lf) did not lead to an increase in nuclear divisions. Instead, the $z f p-1$ mutation suppressed extra nuclear divisions associated with the lin-35; dcr-1, lin-35; alg-1 (Fig. 2A) and lin-35; rde-1 double mutant combinations (Fig. $2 B$ ). This suppression by $z f p-1(l f)$ of a phenotype associated with the lack of transcriptional repressors is comparable with its suppression of a multivulva phenotype $(10,11)$. In both cases it is likely that $z f p-1$ function is required for an enhanced expression of the de-repressed target genes.

Because cyclin $\mathrm{E}$ is one of the target genes repressed by LIN-35, we tested whether enhanced expression of cye-1 mRNA in lin-35(lf) worms requires ZFP-1. Indeed, we found that in the lin-35; zfp-1 double mutant strain, the cye-1 mRNA level was reduced as compared with that in lin-35(lf) (Fig. 2C). We did not observe a reduction in cye- 1 mRNA levels in the $z f p-1$ mutant alone, indicating that its activity is not required for normal levels of expression of this gene.

Genes Repressed by lin-35 and Activated by zfp-1. Our genetic studies of $c y e-1$ regulation and published reports $(10,11)$ indicate that $z f p-1$ may act as an activator of LIN-35(Rb)-repressed genes (Fig. 2). However, the microarray results strongly suggest that ZFP-1 and RDE-4 have a direct repressive effect on a number of other targets, which are not regulated by LIN-35. We were interested in identifying an additional group of genes, those oppositely regulated by lin-35 and $z f p-1$, and further selected for up-regulated expression in lin-35(lf) background and down-regulated expression in the $z f p-1$ mutant with a change in expression intermediate between $z f p-1$ and lin-35 in rde-4(lf) and alg-1(lf) (see SI Text). Fifty-seven genes with expression profiles showing high similarity to this "custom expression profile" were identified (Dataset S1 and Fig. 1). Notably, three Argonaute genes were found in this group. This representation is statistically significant (enrichment factor $38 \times$, $P$ value $6.36 \times 10^{-5}$ ).

We used quantitative real-time PCR to analyze the expression levels of the candidate genes with the largest differences in expression between $z f p-1$ and lin-35 mutants (down-regulated in $z f p-1$ and up-regulated in lin-35) or genes with smaller expression changes that we find interesting, such as Argonaute gene csr-1 (1). The expression of these chosen genes was tested in mutants used for the array analysis and in lin-35; rde-4 and lin-35; zfp-1 double mutants that have limited viability (Fig. $3 A-F$ ). A few genes showed suppression of their enhanced expression in lin-35(lf) background
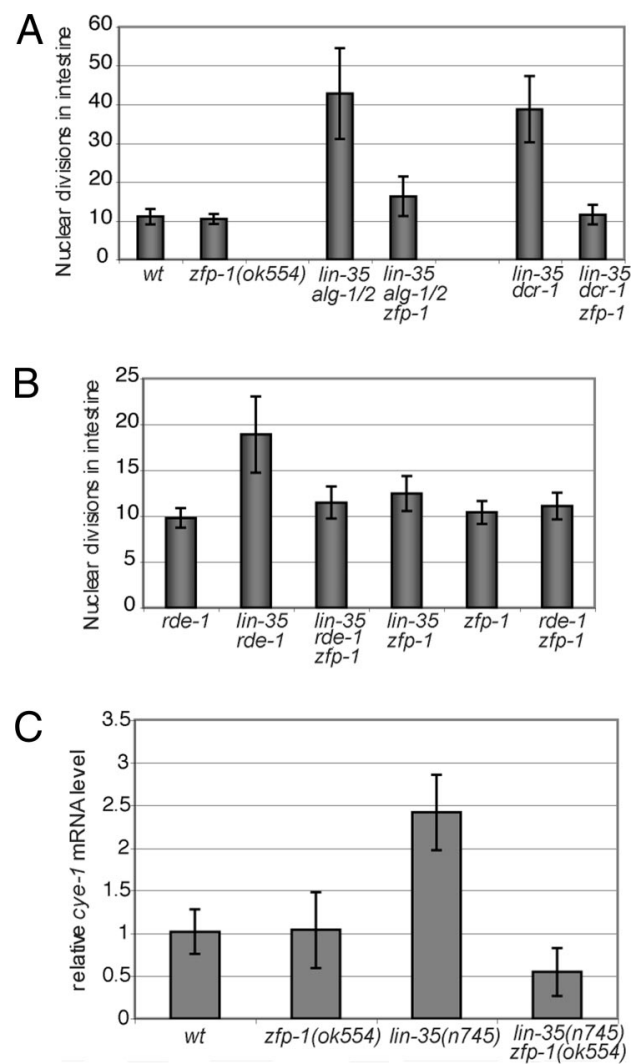

Fig. 2. Suppression of the increased nuclear division phenotype and increased cye-1 mRNA expression in lin-35 mutants by zfp-1(ok554). ( $A$ and $B$ ) Quantification of postembryonic nuclear divisions in the intestine (number of nuclei in adult worms after subtraction of 20 nuclei present in L1) in different genetic backgrounds. Intestinal nuclei were counted in 15-30 worms and data for each genotype are presented as a mean number \pm SD. The following mutants were used lin-35(n745)unc-13(e1091), zfp-1(ok554), rde-1(ne300), alg-1/2(RNAi), and dcr-1(RNAi). Similar results to those shown in $A$ were obtained with lin-35(RNAi) and zfp-1(RNAi). (C) Real-time RT-PCR analysis of the expression levels of cye-1 mRNA in different mutant backgrounds. Levels of cye-1 mRNA were normalized to ama-1 mRNA levels. Results of 2 independent experiments are shown as means and ranges of relative expression compared with wild type. 

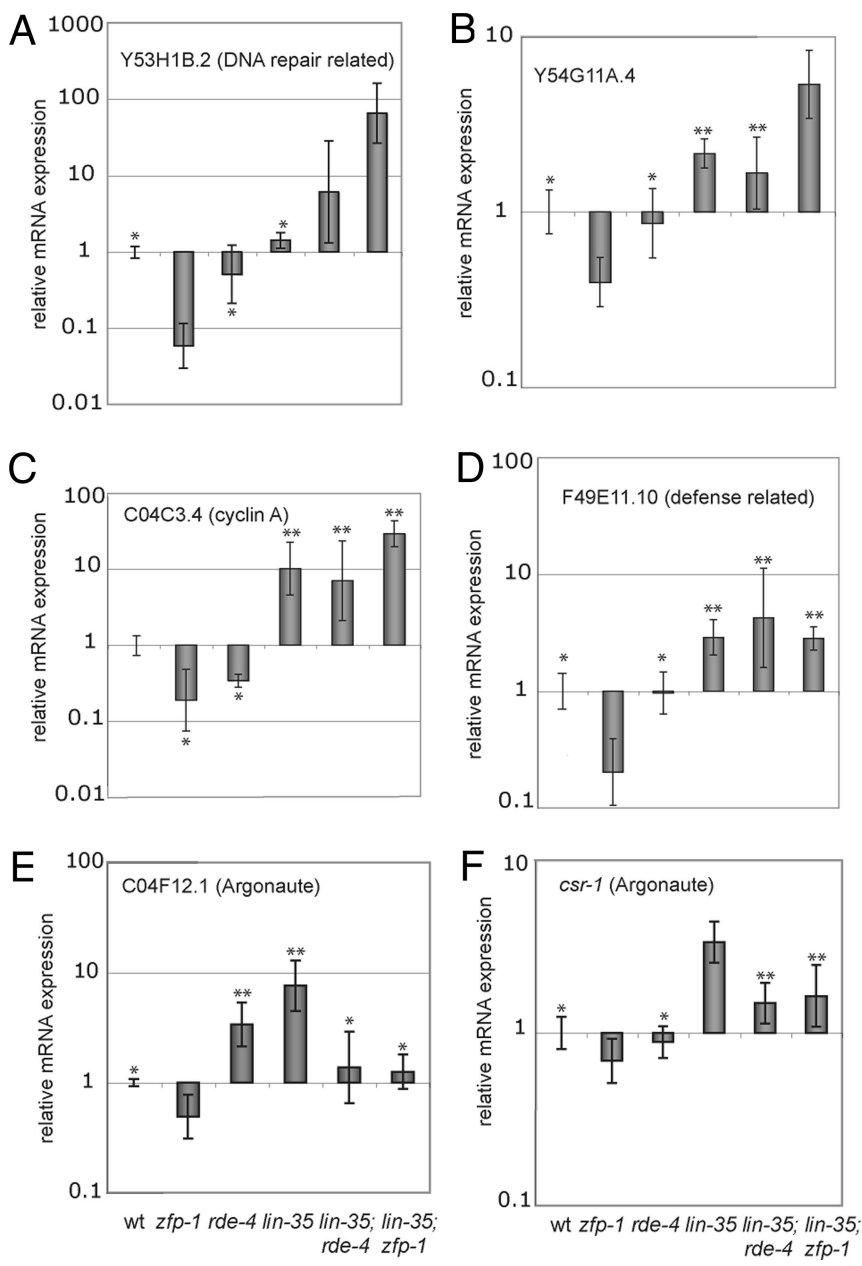

Fig. 3. ZFP-1 acts as a positive and negative regulator of genes repressed by LIN-35. Real-time RT-PCR analysis of the expression levels of indicated LIN-35 and ZFP-1 target mRNAs in different mutant backgrounds. $(A-F)$ Examples of genes up-regulated in lin-35 (-l-) and down-regulated in zfp-1 (-l-). The order of mutants tested is presented the same in all images and is indicated in the bottom. The levels of tested mRNAs were normalized to arx- 2 mRNA levels. Results of 3 RT-PCR experiments are shown as means and ranges of relative expression compared with wild type. Groups of bars labeled * or ** in each image are not statistically different from each other, considering $P<0.05$.

when the $z f p-1$ mutation was added to the lin-35 mutant (Fig. $3 E$ and $F$ ), whereas enhanced expression of other genes was not suppressed by $z f p-1(l f)$ (Fig. $3 A-D$ ). These results reveal a complex regulation of tested genes by LIN-35 and ZFP-1 and suggest that ZFP-1 may have a dual role (of an activator and repressor) in regulating expression of specific targets.

We also performed real time RT-PCR analysis of the expression of several germline-enriched genes with matching siRNAs and repressed by lin-35, but not affected by $z f p-1$ (ok554), similarly to cye-1 (Fig. S3). The lin-35; rde-4 and lin-35; zfp-1 double mutants were also included in this analysis. Loss of $r d e-4$ or $z f p-1$ does not appear to contribute very significantly to the dramatic de-repression of these targets in the lin-35 mutant background.

\section{Discussion}

Our microarray study was motivated by the finding of cooperation between RNAi-TGS and Rb in cyclin E regulation (7). We aimed at identifying more targets of these repressive pathways.

The profile of genes up-regulated in the lin-35 mutant larvae confirms its role in the repression of germline-specific fates in somatic cells (11). More than half of genes up-regulated in the lin-35 mutant have matching endogenous siRNAs (311/535, enrichment factor $2.3 \times, P$ value $2 \times 10^{-60}$ ). Although we cannot exclude a possibility that these endo-siRNAs are produced in the germ line and also function in this tissue, it is equally possible that siRNAs generated in the germ line by RdRP are inherited and function along with LIN-35 to repress germline genes in the soma. Cyclin E is an example of a 'germline-enriched' gene repressed by LIN-35 and RNAi in the somatic tissues (7). This pattern of expression of Cyclin $\mathrm{E}$ is not unique to nematodes. Cyclin $\mathrm{E}$ expression has been shown to be continuous throughout the cell cycle in germline stem cells of Drosophila (23) and embryonic stem (ES) cells from mouse (24) and primates (25). High level of cyclin E was proposed to indicate "stemness" of the cells (23). In somatic cells in these organisms, constitutive Cyclin E expression is repressed with the onset of cell-cycle dependent regulation. Our results demonstrating repression of cyclin $\mathrm{E}$ in the soma along with other germline genes are consistent with this idea.

Interestingly, we found that many RNAi-related Argonaute genes (ppw-1, sago-2, C16C10.3, C04F12.1, and csr-1) are repressed by LIN-35. Argonaute proteins interact with siRNAs and are essential for the silencing process. C. elegans Argonaute genes $p p w-1$, sago-2, and C04F12.1 function redundantly in the RNAi process (1). The level of expression of these genes is elevated eight to ten fold in lin-35(lf) larvae. This finding may explain why the lin-35 mutant is more susceptible to exogenous RNAi $(11,15)$.

We identified very significant enrichment of endo-siRNA target genes among genes up-regulated in $r d e-4\left(P\right.$ value $\left.9.6 \times 10^{-18}\right)$ and $z f p-1\left(P\right.$ value $\left.1.4 \times 10^{-22}\right)$ mutants. Also, these mutants affected a large number of common genes. Previous studies aimed at identifying common misregulated targets among various endoRNAi pathway mutants (3) have not detected large overlaps in misregulated genes or common functional signatures predicting biological pathways where regulation by endogenous RNAi may take place. Therefore, this is the first study demonstrating a connection between $z f p-1$ function and endogenous RNAi processes and identifying specific genes that are 1) endo-siRNA targets, 2) up-regulated in $r d e-4(-/-)$, and 3) up-regulated in $z f p-1(-/-)$ and belong to very specific functional groups, such as regulation of protein translation and germline function (Dataset S2).

We infer that genes commonly up-regulated in the $r d e-4$ and $z f p-1$ mutants and containing matching siRNAs are the direct targets of nuclear RNAi. This prediction is based on the role of $r d e-4$ and $z f p-1$ genes in our characterized system of transcriptional silencing of a transgene (6), the demonstrated requirement of RDE-4 for production of at least some endo-siRNAs $(3,26)$ and on the predicted nuclear function of the ZFP-1 protein. ZFP-1 is a homolog of mammalian protein AF10, which causes myeloid leukemia when fused to MLL (27). Both ZFP-1 and AF10 contain two N-terminal $\mathrm{PHD}$ zinc fingers and a $\mathrm{C}$-terminal leucine zipper domain. Some PHD zinc fingers were recently recognized as histone-binding modules interacting with either methylated $(28,29)$ or unmethylated (30) lysine 4 of histone H3. The protein sequences of most terminal PHD fingers of ZFP-1 and AF10 align very well with histone-binding PHD fingers of other proteins, strongly suggesting that these proteins interact with chromatin via PHD domains. AF10 was shown to recruit histone H3 lysine 79 Dot1 methyltransferase via its leucine zipper domain (31) and to play a role in transcriptional elongation (32). It is possible that ZFP-1 binds histones with its N-terminal PHD domain and recruits different protein factors with its C-terminal domain. It could serve as an adaptor for both activators (Dot1) and repressors (RNAi factors) and regulate gene expression at the transcription elongation step.

The majority of the endo-siRNAs in $C$. elegans is antisense to mature mRNA sequences and is likely produced by RdRPs by using those mature RNAs as templates (2-5). A very recent discovery of an Argonaute protein NRDE-3 that binds endo-siRNAs and shuttles between the cytoplasm and the nucleus (26) further supports a 
possibility that endo-siRNAs and ZFP-1 may work together in the nuclear RNAi pathway in C. elegans.

\section{Materials and Methods}

C. elegans Strains. Worms were maintained on nematode growth medium plates seeded with OP50 bacteria. The strains used are listed in the SI Text. Adult or L4 worms were used for counting intestinal nuclei in strains containing elt-2::gfp/LacZ reporter. RNAi by feeding was performed as described (7). We used lin-35(n745) mutant linked to the weak unc-13(e1091) allele in our experiments to facilitate gene expression comparison between a lin-35 single mutant and lin-35; rde-4 and lin-35; zfp-1 double mutants constructed in unc-13(e1091) background. Only one of eighteen lin-35unc-13-dependent genes that we tested by real-time RT-PCR, sod-3, had an increased expression in unc-13(e1091) background compared with wild type (data not shown). However, its expression was even higher in the lin-35unc-13 strain. Because the functional categories of genes up-regulated in lin-35 mutant were almost identical between our study and that of Kirienko and Fay (22) (Fig. 1), which used an unmarked lin-35 mutant, we believe that the number of false positives in our study, due to unc-13, is very low.

C. elegans Collection for Microarray Experiments. Nematodes were synchronized at L1 stage by hypochlorite treatment of gravid hermaphrodites and hatching their eggs overnight in liquid culture without food. Resulting populations were cultured on OP50 bacteria for $6-7 \mathrm{~h}$ and collected for RNA preparations.

RNA Preparation and Microarray Hybridization. Tri Reagent (MRC) was used for total RNA preparation from frozen worms resulting in 5-30 $\mu \mathrm{g}$ RNA per sample. The quality of RNA samples was confirmed by BioRad Bioanalyzer. Affymetrix GeneChip C. elegans Genome Arrays with a total of 22,625 probesets were hybridized with CDNA and scanned according to manufacturer's standard protocol. All conditions (WT and 4 mutants) were profiled in triplicate. Replicates were biological replicates (separately grown worm populations), with two exceptions: because of shortage of biological material, there were only two bio-

1. Yigit $\mathrm{E}$, et al. (2006) Analysis of the C. elegans Argonaute family reveals that distinct Argonautes act sequentially during RNAi. Cell 127:747-757.

2. Ambros V, et al. (2003) MicroRNAs and other tiny endogenous RNAs in C. elegans. Curr Biol 13:807-818.

3. Lee RC, Hammell CM, Ambros V (2006) Interacting endogenous and exogenous RNAi pathways in Caenorhabditis elegans. RNA 12:589-597.

4. Pak J, Fire A (2007) Distinct populations of primary and secondary effectors during RNAi in C. elegans. Science 315:241-244.

5. Ruby JG, et al. (2006) Large-scale sequencing reveals 21U-RNAs and additional microRNAs and endogenous siRNAs in C. elegans. Cell 127:1193-1207.

6. Grishok A, Sinskey JL, Sharp PA (2005) Transcriptional silencing of a transgene by RNAi in the soma of C. elegans. Genes Dev 19:683-696.

7. Grishok A, Sharp PA (2005) Negative regulation of nuclear divisions in Caenorhabditis elegans by retinoblastoma and RNA interference-related genes. Proc Natl Acad Sci USA 102:17360-17365.

8. Dudley NR, Labbe JC, Goldstein B (2002) Using RNA interference to identify genes required for RNA interference. Proc Natl Acad Sci USA 99:4191-4196.

9. Kim JK, et al. (2005) Functional genomic analysis of RNA interference in C. elegans. Science 308:1164-1167.

10. Cui M, Kim EB, Han M (2006) Diverse chromatin remodeling genes antagonize the $\mathrm{Rb}$-involved SynMuv pathways in C. elegans. PLoS Genet 2:e74.

11. Wang D, et al. (2005) Somatic misexpression of germline P granules and enhanced RNA interference in retinoblastoma pathway mutants. Nature 436:593-597.

12. Wang D, Ruvkun G (2004) Regulation of Caenorhabditis elegans RNA interference by the daf-2 insulin stress and longevity signaling pathway. Cold Spring Harb Symp Quant Biol 69:429-431.

13. Cui $M$, et al. (2006) SynMuv genes redundantly inhibit lin-3/EGF expression to prevent inappropriate vulval induction in C. elegans. Dev Cell 10:667-672.

14. Fay DS, Yochem J (2007) The SynMuv genes of Caenorhabditis elegans in vulval development and beyond. Dev Biol 306:1-9.

15. Lehner B, et al. (2006) Loss of LIN-35, the Caenorhabditis elegans ortholog of the tumor suppressor p105Rb, results in enhanced RNA interference. Genome Biol 7:R4.

16. Tabara H, Yigit E, Siomi H, Mello CC (2002) The dsRNA binding protein RDE-4 interacts with RDE-1, DCR-1, and a DExH-box helicase to direct RNAi in C. elegans. Cell 109:861871.

17. Tabara $\mathrm{H}$, et al. (1999) The rde-1 gene, RNA interference, and transposon silencing in C. elegans. Cell 99:123-132. logical replicates available for the lin-35(n745) unc-13(e1091) I and zfp-1(ok554) III strains; for both, one biological sample was hybridized twice to set up a consistent triplicate structure across the dataset. Subsequent analysis of the replicate structure by using unsupervised hierarchical clustering showed that the agreements between technical replicates is in the same range as those between biological replicates, validating the approach taken. Raw data processing and normalization was performed by using the Bioconductor (33); http://www.bioconductor.org/) packages 'affy' and 'gcrma' to generate the dataset of GC-RMA expression measures (34) used for further analysis.

Data Analysis: Sets of Differentially Expressed Genes. Sets of probesets with upor down-regulated expression in the mutants relative to WT were determined via $t$ test (two-tailed, homoscedastic) with a $P$ value cutoff of 0.01 , requiring in addition an average expression difference of 1.5 or greater on the natural scale.

Complete data analysis description, which includes generation of idealized expression profile, gene assignment and mapping, topomap assignments and graphic generation, is presented in SI Text.

RT and quantitative real time PCR was performed as described in refs. 6 and 7 .

ACKNOWLEDGMENTS. We thank Manlin Luo for performing CDNA labeling and microarray hybridizations and Charlie Whittaker for help with microarray data processing. We also thank Iva Greenwald, Oliver Hobert, Joel Neilson, and Anthony Leung for comments on the manuscript. The elt-2::gfp/lacZ strain was generated by Anne Hart (Massachusetts General Hospital). The zfp-1(ok554) strain was provided by the $C$. elegans Gene Knockout Project at Oklahoma Medical Research Foundation, which is part of the International C. elegans Gene Knockout Consortium. Some strains used in this study were obtained from the Caenorhabditis Genetics Center, which is funded by National Institutes of Health National Center for Research Resources. This work was supported by a Leukemia and Lymphoma Foundation Fellowship \#3260-07 (to A.G.), United States Public Health Service Grant PO1-CA42063 from the National Cancer Institute (to P.A.S.), and partially by Cancer Center Support Grant P30-CA14051 from the National Cancer Institute.

18. Lu X, Horvitz HR (1998) lin-35 and lin-53, two genes that antagonize a C. elegans Ras pathway, encode proteins similar to $\mathrm{Rb}$ and its binding protein RbAp48. Cell 95:981991.

19. Kim SK, et al. (2001) A gene expression map for Caenorhabditis elegans. Science 293:2087-2092.

20. Welker NC, Habig JW, Bass BL (2007) Genes misregulated in C. elegans deficient in Dicer, RDE-4, or RDE-1 are enriched for innate immunity genes. RNA 13:1090-1102.

21. Parrish S, Fire A (2001) Distinct roles for RDE-1 and RDE-4 during RNA interference in Caenorhabditis elegans. RNA 7:1397-1402.

22. Kirienko NV, Fay DS (2007) Transcriptome profiling of the C. elegans Rb ortholog reveals diverse developmental roles. Dev Biol 305:674-684

23. Hsu HJ, LaFever L, Drummond-Barbosa D (2008) Diet controls normal and tumorous germline stem cells via insulin-dependent and -independent mechanisms in Drosophila. Dev Biol 313:700-712.

24. Stead E, et al. (2002) Pluripotent cell division cycles are driven by ectopic Cdk2, cyclin $\mathrm{A} / \mathrm{E}$ and $\mathrm{E} 2 \mathrm{~F}$ activities. Oncogene 21:8320-8333.

25. Fluckiger AC, et al. (2006) Cell cycle features of primate embryonic stem cells. Stem Cells 24:547-556

26. Guang S, et al. (2008) An Argonaute transports siRNAs from the cytoplasm to the nucleus. Science 321:537-541.

27. Daser A, Rabbitts TH (2005) The versatile mixed lineage leukaemia gene MLL and its many associations in leukaemogenesis. Semin Cancer Biol 15:175-188.

28. Li H, et al. (2006) Molecular basis for site-specific read-out of histone H3K4me3 by the BPTF PHD finger of NURF. Nature 442:91-95.

29. Pena PV, et al. (2006) Molecular mechanism of histone H3K4me3 recognition by plant homeodomain of ING2. Nature 442:100-103.

30. Lan F, et al. (2007) Recognition of unmethylated histone H3 lysine 4 links BHC80 to LSD1-mediated gene repression. Nature 448:718-722.

31. Okada Y, et al. (2005) hDOT1L links histone methylation to leukemogenesis. Cell 121:167-178.

32. Bitoun E, Oliver PL, Davies KE (2007) The mixed-lineage leukemia fusion partner AF4 stimulates RNA polymerase II transcriptional elongation and mediates coordinated chromatin remodeling. Hum Mol Genet 16:92-106.

33. Gentleman RC, et al. (2004) Bioconductor: Open software development for computational biology and bioinformatics. Genome Biol 5:R80.

34. Wu Z, et al. (2004) A model-based background adjustment for oligonucleotide expression arrays. J Am Stat Assoc 99:909-917. 